\title{
On the results of the aeropalinospore monitoring of urban air: some features of inside and inter-seasonal variability of lower fungi spores level
}

\author{
Natalya Vlasova $^{1, *}$, Ksenia Mazokha ${ }^{2}$, Lyudmila Kavelenova ${ }^{1}$ and Marina Manzhos ${ }^{2}$ \\ ${ }^{1}$ Samara National Research University, Samara city, 443086 Moskovskoye Shosse 36, Russian \\ Federation \\ ${ }^{2}$ Samara Medical Institute Reaviz, Samara city, 443001 Chapaevskaya street 227, Russian Federation
}

\begin{abstract}
Public health risks associated with molds include sensitization, allergic symptoms, irritation and/or toxicity. Sensitization to fungal allergens often occurs in patients who are hypersensitive to other groups allergens, including plant pollen. Both plants and molds are characterized by active growth in warm seasons, in temperate latitudes from April to October, which determines the presence of pollen grains of plants and mold spores in the atmospheric air during this period. This determines the needs in monitoring activity aimed to biogenic pollutants evaluation in the air of large cities where many people live compactly. The report presents the monitoring results of Alternaria, Cladosporium and other lower fungi spores in the air of Samara city in 2015 - 2020. It has been established that for the air environment of Samara, lower fungi cannot be considered as the predominant source of allergens. The high concentration of fungal spores is most often caused by fungi of Alternaria genus, which leads to the development of sensitization in $27.7 \%$ of children and $14.6 \%$ of adults in the region. The results of aeropalinospore monitoring demonstrated the lesser significance of Cladosporium genus fungi as a risk factor, or an allergens source for Samara city (Middle Volga region).
\end{abstract}

\section{Introduction}

Of the more than 100,000 known fungal species, about 400 are listed as potential and/or confirmed sources of allergens whereas the WHO Allergen Database contains 111 fungal allergens from 29 fungal species [1]. If higher fungi usually develop on a dense substrate and release only mature spores into the air, mold fungi and yeast supply both mycelium fragments and spores into the air, that is why they become the most common components

\footnotetext{
*Corresponding author: nwlasova@mail.ru
} 
of air plankton [2]. Public health risks associated with molds include sensitization, allergic symptoms, irritation and/or toxicity. Fungi of the Alternaria genus play a leading role in the formation of sensitization in the human population [3]. Nevertheless, although thousands of fungal spores can be detected in standard air samples, the level of sensitization to lower fungi, mainly found in the various rooms air (for example, Aspergillus, Penicillium) or outdoors (Cladosporium, Alternaria), is below 5\% [4].

Among patients with various atopic diseases, the prevalence of sensitization to Alterniria allergens increases and, according to various authors, ranges from 3 to $66 \%$ [5]. Sensitization to fungal allergens often occurs in patients who are hypersensitive to other groups allergens, including plant pollen, the proportion of such patients reaches $75-80 \%$ [6]. Both plants and molds are characterized by an active growth during warm season, in temperate latitudes from April to October, which determines the presence of both plants pollen grains and mold spores in the atmospheric air during this period. These considerations determine the necessivity for monitoring of biogenic pollutants (fungi spores and pollen) presence in the air of large cities where many people live compactly. However, until recently, aeropalinospore monitoring has not become an obligatory part of environmental monitoring in our country. Samara is one of the cities in Russia where such work has been consistently carried by us out since 2015 . Earlier, we published works on the results of pollen monitoring. The frequency and concentration of various lower fungi spores in the air are characterized by seasonal variability. This has been shown for a number of European countries: for example, the seasonal maximum of Alternaria spores for Southern Europe is reached from May to August, for Northern Europe - from July to September; the concentration of Aspergillus, Cladosporium, Penicillium spores usually increases in autumn [6]. For the Middle Volga region, the issue of the seasonal dynamics of allergenic fungal spores in the city air has not been previously considered. Our report presents the results of Alternaria, Cladosporium and other lower fungi spores monitoring in Samara city air in $2015-2020$.

\section{Materials and Methods}

The Samara city is located in the Middle Volga region of Russia, 53011'0 " north latitude, $5008^{\prime} 48$ " east longitude, $100-120 \mathrm{~m}$ above sea level. The specificity of the Samara sity conditions is determined by its ecotonal localization (forest-steppe), as well as by the continental climate with its inherent variability of weather conditions, which has noticeably increased in recent years. Aeropalinospore monitoring was carried out during the growing season from April 1 to September 30. Pollen grains and spores were captured using a slit trap with a standard 2X14 mm inlet (Penza STI Center Fact Sheet No. 51-03) designed for field work. The air was pumped through impactor for 25 minutes at a rate of $10 \mathrm{~L} / \mathrm{min}$, onto glass slides coated with a mixture of petroleum jelly and wax. The impactor was installed at a height of 10 meters above ground level. The operating time of the device was 25 minutes for each glass, a total of three glasses at 8, 14, 20 hours. In the obtained preparations, pollen grains and spores were counted with the subsequent determination of their belonging to various systematic groups [7]. Subsequently, the content of pollen grains in 1 cubic metre was determined. When compiling the pollen calendar, the quantitative content of pollen was averaged. The processing of slides was carried out at the Chair of Ecology, Botany and Nature Protection, Samara University. The data obtained were processed using Microsoft Excel. 


\section{Results and discussion}

The weather features of 2015-2020 growing seasons (Fig. 1.) found noticeable differences in the precipitation amount and rhythm, in the temperature changes dynamics. This corresponded to the formation of arid conditions in different months - moisture deficit of varying severity, sometimes added by increased temperature background (June 2015, JuneAugust 2016, August 2017, May-June and August-September 2018, June-July 2019, July 2020). The humid conditions corresponded to other months, including sharply expressed ones (September 2016, April-June 2017, April 2018). The variability of the spores level in the air depending on the concrete weather seasons has been repeatedly noted for different regions of the world [for example, 8] In such different conditions in Samara, the rate and intensity of lower fungi development, the spores formed number, and the duration of their stay in the air changed accordingly.

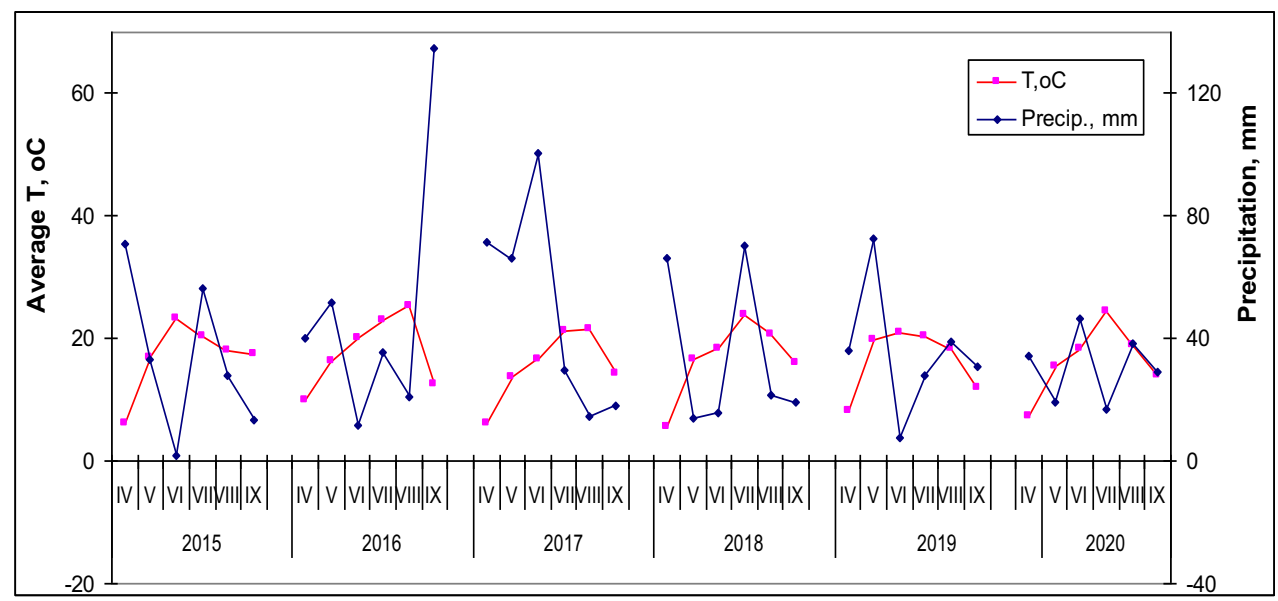

Fig. 1. Values of environmental parameters in 2015-2020 vegetation seasons, Samara.

Analyzing the seasonal dynamics of Alternaria, Cladosporium, as well as other lower fungi spores in the Samara city air for the 2015-2020 growing seasons (Fig. 2.), we can note both quantitative differences and certain shifts in dynamics. So, 2017 season showed the minimum levels of all detected spores groups, which can be explained by the abundance of precipitation and a relatively low temperature background in the first half of the growing season. The weather conditions specificity in 2015 provoked an abnormally high, subsequently not observed, Cladosporium spores level and an increased Alternaria spores level in September. The similarity features in relation to the spore component of the air environment of Samara city in the studied periods can be considered a higher, in comparison with Cladosporium and Alternaria, level of other lower fungi spores, as well as the confinement of the seasonal peak concentration of Cladosporium and Alternaria spores to August (in most cases) or by July (spores of other lower fungi). 


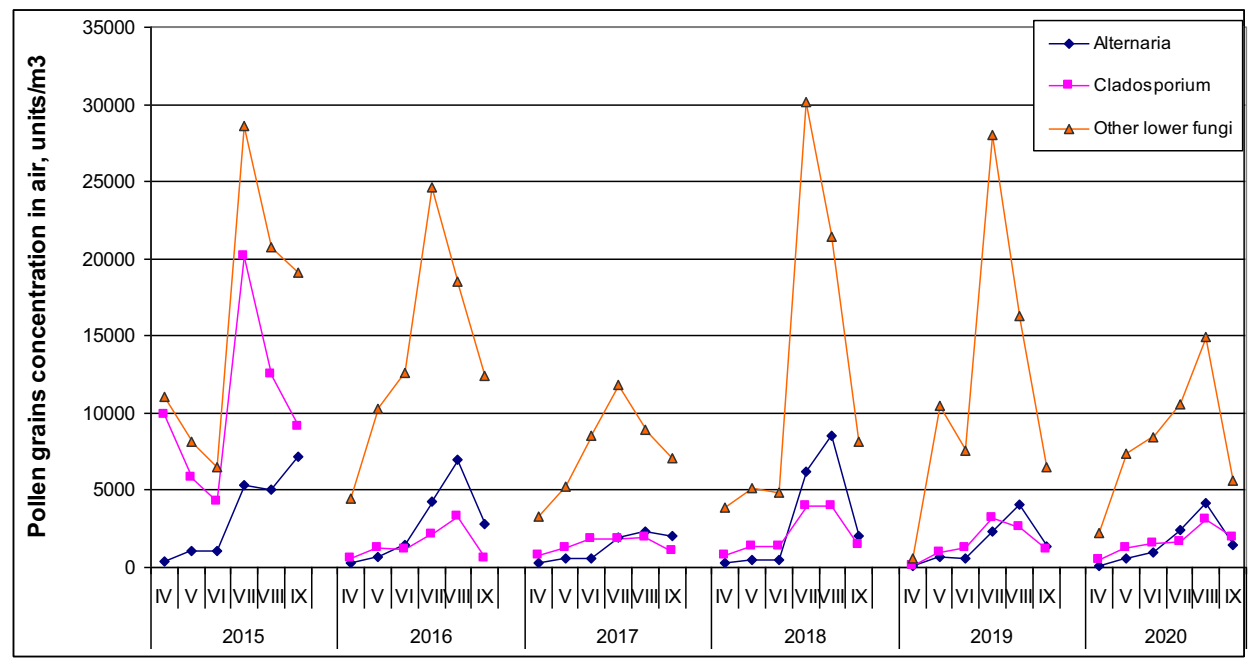

Fig. 2. Features of the seasonal dynamics of the concentration of spores of lower fungi in the air of Samara, 2015-2020

The duration of periods with different fungal spores levels in the urban air was analyzed. For this expert assessment, we have identified conditional concentration ranges corresponding to different classes - from virtually none to the maximum detected level of spores in the air. The number of days in which air quality corresponded to one of the conditional classes was determined for each of the seasons (2015 - 2020) (Fig. 3). Analyzing the growing seasons in accordance with the number of days when different levels of Cladosporium, Alternaria or other lower fungi spores were observed, it can be noted that, with the same duration of observation seasons (183 days), the picture for different years and fungi groups was different.

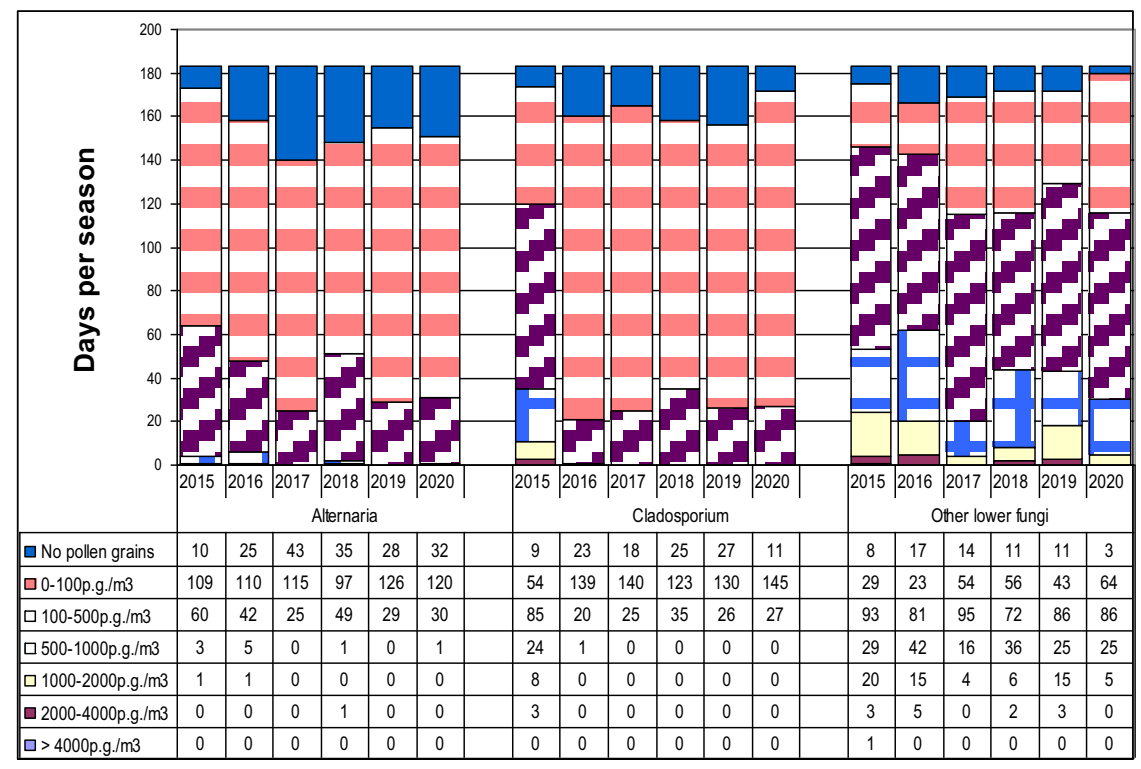

Fig. 3. Duration of periods with different levels of spores of lower fungi in the air of Samara city during the growing seasons of 2015-2020 
Thus, there was a noticeable difference in the number of days when no Alternaria spores were detected in air samples - from 10 to 43 ; more similar was the number of days when the concentration of Alternaria spores did not exceed 100 units per square meter of air from 97 to 126. In accordance with the previously established threshold value for Western Europe (100 spores $\left./ \mathrm{m}^{3}\right)$ [9], these periods can be regarded as "safe", and days with a dangerous level of Alternaria spores, depending on the year, form a period of 30 (2017) to 60 days (2017). This is consistent with the results of clinical and allergological studies, which confirm the high etiological significance of Alternaria genus fungi in the development of respiratory allergy in Samara. The prevalence of sensitization to Alternaria in the region is $27.7 \%$ among children and $14.6 \%$ among adults. Combined sensitization to Alternaria and pollen allergens was generally diagnosed in $14 \%$ of patients [10].

The threshold level for Cladosporium (3000 spores $\left./ \mathrm{m}^{3}\right)$ indicated in the literature [9] was exceeded only in 2015, the number of such days was 3. The years 2016-2020 were characterized by the predominance of periods when up to 100 spores $/ \mathrm{m}^{3}$ were detected in the air of Samara (123-140 days). More variable was the duration of the periods of spores absence (from 11 to 27 days) and their detection at a concentration of 500-1000 spores $/ \mathrm{m}^{3}$ (from 20 to 35 days), which corresponded to a change in the duration of these time intervals of 1.75-2.7 times.

\section{Conclusion}

Thus, for the air of the city of Samara, lower fungi cannot be considered as the predominant source of allergens. Primary data characterizing fungal sensitization in patients were obtained by diagnostic determination of the level of IgE antibodies to fungal allergens by immunoblotting (RIDA Allergyscreen, R-Biopharm, Germany) and used in connection with monitoring data. It can be noted that a high concentration of fungal spores in the air is most often caused by fungi of the genus Alternaria, which leads to the development of sensitization in $27.7 \%$ of children and $14.6 \%$ of adults in the region. The results of our aeropalinospore monitoring also showed much lesser importance of Cladosporium spores as a risk factor (a source of allergens) for the conditions of the city of Samara (Middle Volga region).

\section{References}

1. Nomenclature of allergens [Electronic resource]. http//www.allergen.org (15.01.2021)

2. R. Crameri, M. Garbani, C. Rhyner, C.Huitema, Aller. 69, 176 (2014)

3. E. Levetin, E. Horner, A. Scott, J. of Aller.and Clin.Immunol. 4(3), 375 (2016)

4. R. Schmitz, U. Ellert, M. Kalcklosch, S. Dahm, M. Thamm, Int. Arch. Aller.Immunol. 162, 263 (2013)

5. B. Simon-Nobbe, U. Denk, V. Poll, R. et al. Int. Arch. Aller.Immunol. 145 (1), 58 (2008)

6. K. Reijula, M. Leino, H. Mussalo-Rauhamaa, Aller.Asth.Immunol. 91 (3), 280 (2003)

7. Principles and methods of aeropalynological studies. (Medicine, 1999) (in Russian)

8. M. Syed, J. Envir.Monit. 14 (3),1006 (2012)

9. Y. Olsen, C. Ambelas Skjøth, O. Hertel, K. Rasmussen, Aerobiol. 36,141 (2020)

10. K. Masokha, M. Manzhos, L. Khabibulina, L. Aseeva, A. Seleznev, Rus. J. Immunol. 23 (4), 461 (2020) 